\title{
Loss of Transgene following ex vivo Gene Transfer is Associated with a Dominant Th2 Response: Implications for Cutaneous Gene Therapy
}

\author{
Zhenmei Lu ${ }^{1}$ and Soosan Ghazizadeh ${ }^{1,2}$ \\ ${ }^{1}$ Department of Dermatology, College of Physicians and Surgeons, Columbia University, New York, \\ New York, USA \\ ${ }^{2}$ Department of Oral Biology and Pathology, School of Dental Medicine, Stony Brook University, \\ Stony Brook, New York, USA
}

\begin{abstract}
Host responses to therapeutic gene products are potentially serious complications in cutaneous gene therapy. Controlling immune responses to the thera-peutic antigen may therefore be critical for an effective therapy. Both ex vivo and in vivo gene transfer to epidermal stem cells has been shown to induce transgene-specific immune responses; however, whether the mechanism of immune activation is the same is not clear. In this study, we have characterized transgene-specific immune responses in an ex vivo model of epidermal gene transfer using green fluorescent protein as a model antigen and retrovirus-mediated gene delivery. Contrary to T helper (Th)1-type responses induced following in vivo gene transfer to epidermis, rejection of ex vivo-transduced keratinocytes was associated with Th2/eosinophilc responses. These responses were characterized by interleukin (IL)- 4 and IL-5 production by T cells, a predominance of anti-green fluorescent protein IgG1 in serum, the presence of numerous eosinophils within rejected skin, and a lack of class I-restricted cytotoxic $\mathrm{T}$ lymphocyte response. Pretreatment of mice receiving ex vivo transduced keratinocytes with neutralizing anti-IL-5 antibody prevented eosinophil infiltration and prolonged survival of transduced epidermis. These data indicate a role for the Th2/eosinophilic pathway in rejection of $e x$ vivo-transduced keratinocytes, suggesting different requirements for achieving tolerance for $e x$ vivo and in vivo approaches to gene therapy.
\end{abstract}

\section{INTRODUCTION}

For a number of indications in which gene transfer is a possible approach to therapy, two basic methods of gene delivery are being considered, ex vivo or in vivo. ex vivo transfer entails gene delivery to cells or tissues outside the body and has several attractive features, including the ability to reduce or eliminate exposure of the host to vector as well as the possibility of verifying expression before engraftment back into the host. However, for a number of targets of gene transfer, in vivo is the only option as ex vivo tissue maintenance and transplantation are not possible or feasible.

Efficient gene transfer to the epidermis has been accomplished by using recombinant retroviral and lentiviral vectors as a vehicle in both ex vivo and in vivo models of cutaneous gene transfer.

(c) The American Society of Gene Therapy

Correspondence: Soosan Ghazizadeh, Department of Oral Biology and Pathology, Stony Brook University, New York, New York 11794-8702, USA. sghazizadeh@notes.cc.sunysb.edu. 
${ }^{1-3}$ Regardless of the method of gene transfer however, expression of a neoantigen in nontolerant hosts has been shown to be associated with immunological rejection of the genetically, modified epidermis and the appearance of host antigen-specific immune responses. ${ }^{4,5}$ However, whether the mechanism of immune rejection is the same following ex vivo and in vivo gene transfer is not clear.

Using in vivo transduction of mouse skin with retroviral vectors as a model to delineate the nature of immune responses in cutaneous gene transfer, we identified transgene products as the antigen responsible for the rejection of transduced cells. Expression of an antigenic transgene product in the skin by in vivo gene transfer resulted in the generation of $\mathrm{T}$ helper (Th)1-type immunity including T-cell cytotoxicity mediated mainly by $\mathrm{CD} 8^{+} \mathrm{T}$ cells. Moreover, blockade of both $\mathrm{CD} 4^{+}$and $\mathrm{CD} 8^{+} \mathrm{T}$ cells was required for long-term transgene expression. ${ }^{4}$ Induction of humoral and cellular responses to a transgene product requires antigen-presentation by bone marrow-derived professional antigen presenting cells. ${ }^{6}$ Direct injection of retroviral particles likely results in direct transduction of antigenpresenting cells and activation of $\mathrm{T}$ cells by the classical endogenous major histocompatability complex class I pathway, although cross-presentation of transgene products expressed in keratinocytes by antigen-presenting cells is also possible. ${ }^{7-9}$ Moreover, recombinant viral particles could activate innate immunity and provide the danger signal required for activation of adaptive immunity. ${ }^{10,11}$

Ex vivo gene transfer is thought to provide a safer and less immunogenic approach than in vivo gene transfer. ${ }^{12}$ Activation signals provided to the immune system following in vivo administration of recombinant viral particles are avoided in ex vivo gene transfer and antigen presentation and priming of cytotoxic T lymphocyte (CTL) cells is restricted to crosspresentation. We recently developed a murine model of ex vivo gene transfer targeted to keratinocytes in immunocompetent mice and showed that engraftment of ex vivo-transduced or transgenic keratinocytes expressing a neoantigen resulted in induction of transgene-specific immune responses and clearance of genetically modified keratinocytes. However, in mice tolerant to the transgene product, transduced keratinocytes persisted for more than 40 weeks. ${ }^{5}$ The induction of immune response in this model was likely due to cross-presentation of keratinocyte-derived antigens. The release of proinflammatory cytokines and apoptotic cellular material that are produced along with transgene product during the initial stages of transplantation provided proinflammatory signals necessary for the induction of transgenespecific immunity to keratinocyte-derived neoantigens. ${ }^{13,14}$ Although this study reiterated the immunogenicity of transgene product as a major limitation in cutaneous gene therapy, the mechanism responsible for rejection of transgenic keratinocytes in the ex vivo method of gene transfer was not characterized.

If the goal in developing effective gene therapies is to ensure persistent expression of the therapeutic gene through suppression of unwanted responses or induction of tolerance, it would be vital to understand if the mechanism of immune rejection differs following ex vivo or in vivo gene transfer. Effector mechanisms involved in the rejection of antigen-bearing cells are influenced by the pathway of antigen presentation and differentiation of Th cells into Th1 or Th2 phenotypes. ${ }^{15}$ Accordingly, the requirements for achieving tolerance or hyporesponsiveness are likely to differ. The aim of this study was to delineate the effector pathways involved in the rejection of ex vivo-transduced keratinocytes in order to develop effective strategies for diverting destructive immune responses and achieving an effective clinical cutaneous gene therapy. Our analysis underscores the differences between immune responses to in vivo and ex vivo cutaneous gene transfer and identifies eosinophils as key effector cells in the rejection of ex vivo-transduced keratinocytes. 


\section{RESULTS}

\section{Kinetics of transgene loss in the ex vivo method of gene transfer to keratinocytes}

To determine the kinetics of transgene loss following transplantation of ex vivo-transduced keratinocytes, primary cultures of newborn FVB mouse keratinocytes were transduced with a retroviral vector encoding green fluorescent protein (GFP), mixed with syngeneic fibroblasts, and grafted to naïve FVB or transgenic FVB mice tolerant to GFP (Gad-GFP), as described previously. ${ }^{5}$ Skin grafts were examined weekly for surface GFP expression and signs of graft rejection. Clinical signs of graft rejection, including erythema and hair loss, were noted at 3 weeks only in FVB mice before a progressive loss of surface GFP. Complete loss of expression was noted between 4 and 5 weeks post-grafting $(n=10)$, whereas in GFP-tolerant mice, no change in GFP expression was noted for the entire period of observation confirming GFP as a dominant rejection antigen (Figure 1a). Analysis of sera collected from FVB mice at 1, 2, 4, 6 , and 10 weeks post-grafting showed GFP-specific antibodies appearing as early as 2 weeks post-transduction and increasing over time to more than $5 \mathrm{mg} / \mathrm{ml}$ (Figure 1b). No antibody was detected in GFP-tolerant mice $(n=6)$. These data confirmed the induction of transgene-specific immune responses to GFP-expressing keratinocyte resulting in complete rejection of $\mathrm{GFP}^{+}$ keratinocytes by 5 weeks post-transplantation.

\section{Tissue infiltration of eosinophils in ex vivo-transduced skin}

To characterize the inflammatory responses to the grafts, transduced skin grafted onto FVB or GFP-tolerant mice were harvested at 4 weeks post-grafting and tissue sections were subjected to histological examination. Transduced skin grafts from FVB mice demonstrated intense inflammation and loss of tissue architecture, including epidermal hypertrophy, hyperkeratosis (thickening of the stratum corneum), and an intense dermal mononuclear cell infiltrate (Figure 2a). A more detailed analysis of this inflammatory infiltrate revealed mast cells in various stages of degranulation (Figure 2b), numerous eosinophils along and within the epidermis and hair follicles (Figure 2d), and mononuclear cells including $\mathrm{CD}^{+}$cells and B cells and a few but significant number of $\mathrm{CD}^{+}$cells (Figure 2c, e, f). On the contrary, GFP-expressing keratinocytes grafted onto transgenic GFP-tolerant mice formed a well-differentiated tissue with no signs of inflammation (data not shown). ${ }^{5}$ The pathology of ex vivo transduced skin in FVB mice during rejection was suggestive of allergic inflammation and significantly different from that seen following in vivo-transduction of skin. Analysis of in vivo-transduced skin at the time of rejection ( 2 weeks post-transduction) demonstrated minimal tissue damage and dominant lymphocytic infiltrates (Figure $2 \mathrm{~g}$ ). Only rarely were eosinophils present within the in vivo-transduced skin; instead, epidermis and hair follicles were infiltrated with a large number of $\mathrm{CD} 8^{+} \mathrm{T}$ cells and $\mathrm{CD} 4^{+} \mathrm{T}$ cells (Figure $2 \mathrm{~h}-\mathrm{i}$ ) supporting a Th1 inflammatory response. The observed histological differences between in vivo- and ex vivo-transduced skin suggested a different effector mechanism for immune rejection of ex vivo-transduced skin.

\section{Analysis of transgene-specific immune responses in ex vivo-transduced mice reveals a Th2 inflammatory response}

Rejection of transduced cells in gene therapy is often associated with Th1-type responses, with a dominant role for $\mathrm{CD} 8^{+}$CTLs. ${ }^{4,16,17}$ Eosinophil-mediated tissue destruction is a hallmark of allergic inflammation and Th2-derived responses. ${ }^{18,19}$ To obtain further insight into the type of immune responses induced by ex vivo gene transfer, GFP-specific cellular and humoral responses were assessed and compared with those of mice transduced by direct injection of a retroviral vector targeted to epidermis (hereafter referred to as in vivo transduced). To restrict GFP expression to keratinocytes following in vivo gene transfer, a transcriptionally targeted retroviral vector in which transgene expression is controlled by the involucrin promoter and targeted to suprabasal layers of epidermis was used. ${ }^{20}$ Despite restriction of transgene products to keratinocytes, GFP-specific immune responses induced following in vivo transduction of 
mouse skin with this vector are comparable to LZRS-GFP (data not shown). Analysis of GFPspecific CTL activity in lymphocytes isolated from ex vivo-transduced mice and re-stimulated in vitro with irradiated GFP-expressing splenocytes revealed no significant cytotoxicity when incubated with GFP-expressing fibroblasts (Figure 3a). On the contrary, significant CTL activity was present in lymphocytes isolated from in vivo-transduced mice (Figure 3a) consistent with Th1-type responses.

Although few in number, $\mathrm{CD} 8^{+} \mathrm{T}$ cells were among the immune cells infiltrating the ex vivotransduced skin, and the absence of CTL activity in vitro may not represent their role in vivo. As immunological memory, the ability of the immune system to respond more strongly to successive exposure to the same antigen, is a hallmark of T-cell-mediated immunity, ${ }^{21}$ we assessed CTL memory in transduced mice. In the presence of CTL memory, rejection of the GFP-expressing epidermal graft is expected to follow a more rapid kinetics. Both in vivo- and ex vivo-transduced mice that have already rejected $\mathrm{GFP}^{+}$keratinocytes were challenged by implantation of $\mathrm{GFP}^{+}$keratinocytes (Figure $3 \mathrm{~b}$ ). Weekly analysis of surface GFP expression demonstrated that in mice transduced by the in vivo method where $\mathrm{Th} 1$ responses were induced, skin grafts were lost in 1 week following transplantation, indicating the presence of GFPspecific CTL memory $(n=4)$. However, in ex vivo-transduced mice, loss of the second graft followed a similar kinetic as the first graft with a progressive loss of surface GFP at 4 weeks post-grafting in the majority of mice $(n=6)$ (Figure $3 \mathrm{~b}$ ). The lack of in vitro and in vivo CTL activity in ex vivo-transduced mice was consistent with Th2-type responses dominated by interleukin (IL)-4, which have been shown to inhibit cell-mediated immunity. ${ }^{22}$

To examine if transplantation of ex vivo-transduced keratinocytes results in Th2 differentiation, the profile of cytokines secreted by $\mathrm{T}$ cells was assessed in a mixed lymphocyte reaction using irradiated GFP-transgenic splenocytes as stimulators. Lymphocytes from the spleen and lymph nodes draining the transduced skin (axillary nodes) were isolated at the time of skin rejection and levels of IL-2, IL-4, IL- 5 and interferon- $\gamma$ secreted by T cells were assessed by cytokinespecific enzyme-linked immunosorbant assay (ELISA). Compared with controls (GFP-tolerant mice), there was more than a five fold increase in the level of IL-4 and a 12-fold increase in the levels of IL-5 secreted by T cells isolated from ex vivo-transduced FVB mice (Figure 4). IL-2 level was also slightly elevated, but there was no significant interferon- $\gamma$ production (Figure 4). This cytokine profile was consistent with type II responses and differed significantly from in vivo-transduced mice in which high levels of interferon- $\gamma$ and suppressed IL-4 production indicated Th1-type responses (Figure 4).

As Th1 cells stimulate production of immunoglobulin (Ig) G2a antibodies and Th2 cells stimulate production of specific IgG1 and IgE antibodies, ${ }^{22}$ the isotypes of anti-GFP antibodies in sera collected at 4 weeks from transduced mice were examined. Consistent with the cytokine profile, analysis of serum immunoglobulins in ex vivo-transduced mice showed increases in both IgE and IgG1 levels with an IgG1:IgG2a ratio of 9:1 (Figure 5). The Ig profile of ex vivo-transduced mice was significantly different from that of in vivo-transduced mice in which IgG2a was predominant with a IgG1:IgG2a ratio of 1:2.5 (Figure 5).

All together, the lack of GFP-specific CTLs, the prevalence of anti-GFP IgG1, the production of anti-GFP IgE, and the elevated levels of IL-4 and IL-5 were consistent with Th2-polarized immune responses and suggested Th2 inflammatory responses as the major effector pathway in the rejection of genetically modified keratinocytes following ex vivo cutaneous gene transfer.

\section{Eosinophils play a major role in the rejection of ex vivo-transduced keratinocytes}

Th2-type cytokines dominated by IL-4 have been shown to induce eosinophil-mediated tissue destruction. ${ }^{18,19}$ Intense eosinophilic infiltrate and significant tissue destruction in ex vivotransduced skin suggested a role for eosinophils. IL-5 plays an essential role in the maturation, 
differentiation, and migration of eosinophils from bone marrow. ${ }^{23} \mathrm{~A}$ single dose of an inhibitory antibody to IL-5 (TRFK-5, $1 \mathrm{mg} / \mathrm{kg}$, intraperitoneally) has been shown to inhibit reversibly the release of eosinophils from bone marrow for at least 8 weeks and inhibit antigendependent lung eosinophilia in mice for at least 12 weeks. ${ }^{24}$ To assess the role of eosinophils in the rejection of transduced keratinocytes, mice were injected intraperitoneally with either 1 $\mathrm{mg} / \mathrm{kg}(30 \mu \mathrm{g})$ of TRFK-5 or a control antibody (Rat IgG) 1 day before transplantation of transduced keratinocytes. Surface GFP expression was monitored and recorded weekly and the results are shown in Figure 6.

As expected, by 4 weeks post-grafting in mice pretreated with Rat IgG, there was a significant reduction in surface GFP expression, indicating rejection of $\mathrm{GFP}^{+}$keratinocytes. In TRFK-5treated mice however, no significant reduction in surface GFP was evident for the first 6 weeks post-grafting $(n=6)$ (Figure 6a). After this time however, there was a gradual loss of GFP ${ }^{+}$ keratinocytes with a complete loss at 10 weeks post-grafting. Analysis of blood smears indicated that IL-5 blockade resulted in suppression of eosinophilia (data not shown), which in turn resulted in a significant reduction in eosinophilic infiltrates (Figure 6b). Histopathology of transduced skin in these mice showed inflammation and epidermal hyperplasia but no sign of tissue necrosis as observed in mice treated with control antibody (Figure 6b). Quantitative analysis of inflammatory cells, including mast cells, B cells, monocytes, $\mathrm{CD} 4^{+}$cells and $\mathrm{CD} 8^{+}$cells at 4 weeks post-grafting, indicated no significant change in their distribution between mice treated with TRFK-5 and control Rat IgG, except for a modest reduction in the number of CD4+ cells in the former (Figure 6c). Despite an intense non-eosinophilic dermal infiltrate and epidermal hyperplasia in TRFK-5-treated mice, transgene expression was significantly prolonged, indicating a dominant role for eosinophils in rejection of transgenic keratinocytes. These data suggest that long-term survival of ex vivo-transduced keratinocytes expressing a neoantigen may require inhibition of $\mathrm{Th} 2$ responses.

\section{DISCUSSION}

Therapeutic gene transfer for patients carrying null mutations in the affected allele will likely induce immune responses resulting in transient transgene expression and failure of the therapy. Understanding the mechanisms underlying activation of immune responses and type of effector cells mediated the transgene loss is important for planning how to avoid or overcome these responses. Preclinical animal models of in vivo gene transfer have indicated a major role for CTL-mediating killing in the rejection of genetically-modified cells. Accordingly, the

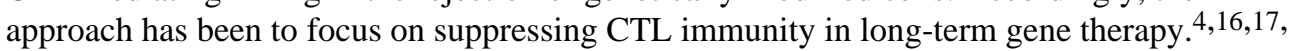
${ }^{25-27}$ However, this study shows that ex vivo gene transfer promotes a dominant Th2/ eosinophilic response, suggesting that a different approach for suppressing destructive responses may be needed.

Orthotopic transplantation of keratinocytes expressing an intracellular antigen induced a Th2biased response highlighted by an increased secretion of IL- 4 and IL- 5 by T lymphocytes, elevated levels of anti-GFP IgG1 and IgE in sera, infiltration of numerous eosinophils and mast cells into the transduced skin, and the lack of in vitro and in vivo CTL activity. Recent evidence suggests that IL-4 and IL-5 released from Th2 cells promote eosinophil-mediated rejection.

${ }^{18}$ Eosinophils are recruited to the graft and exert cytotoxic activity through the release of several molecules such as leukotriene, superoxides, major basic protein, and eosinophil cationic protein. ${ }^{15}$ Intense eosinophilic infiltrate and significant tissue destruction in ex vivotransduced skin suggested that eosinophils were the major effector cells mediating rejection of transgenic keratinocytes. A single dose of a neutralizing antibody to IL-5, the principal eosinophil-activating cytokine, 28 reversibly inhibited transgene-dependent eosinophilic infiltrates in transduced skin for at least 8 weeks, as shown in other experimental models. ${ }^{24}$ Suppression of eosinophilia was sufficient to prevent acute rejection of transduced cells, 
although other immunocytes infiltrated into the transduced tissue during this period. A comparable cytokine and antibody profile in anti-IL-5-treated and control mice (data not shown) indicated that antigen presentation and priming of $\mathrm{T}$ cells were not altered and the effects of IL-5 blockade were specific to eosinophils. It is worth noting that repeated injection of anti-IL-5 antibody at 4 weeks intervals did not prevent the gradual loss of GFP ${ }^{+}$keratinocytes observed after week 6 postgrafting (data not shown), indicating that other effector pathways may mediate rejection of transduced cells in the absence of eosinophils.

Although type 2 immune responses and accumulation of eosinophils are hallmarks of allergic responses (e.g., atopic dermatitis and asthma), recent studies have shown a role for this pathway in skin and heart rejection where donor and recipients differ at major histocompatibility complex class II antigen or when $\beta 2$-microglobulin was used as a minor transplantation antigen. ${ }^{29-31}$ However in these models the effector role of the Th2/eosinophilic pathway became more apparent when both CD8- and CD4-mediated cytotoxicity were not operative. In FVB mice used in our studies, both CD8 and CD4 T cells were functional, and when challenged by direct injection of the same vector into their skin, the mice developed a significant GFP-specific CTL activity and memory. Therefore, our study suggests that the absence of functional CD ${ }^{+} \mathrm{T}$ cells is not a requirement for Th2/eosinophilic-mediated rejection of transplanted cells expressing an antigen.

Several factors have been shown to drive Th2 differentiation including the subset of dendritic cells activated and the cytokines produced by them. ${ }^{32}$ Interestingly, one cytokine recently identified as the initiator of Th2 responses is thymic stromal lymphopoietin, which has been found to be highly expressed by cultured human keratinocytes and induced by vitamin $\mathrm{D}_{3}$ in mouse keratinocytes.33,34 Thymic stromal lymphopoietin transcript was detected in mouse keratinocytes grown under the culture condition used in our studies and its levels were increased significantly in regenerated epidermis by 1 week post-grafting (data not shown). Whether expression of thymic stromal lymphopoietin in implanted cultured keratinocytes drives transgene-specific responses toward $\mathrm{Th} 2$ differentiation and how dependent immune responses induced by ex vivo gene transfer is on the type of cell/tissue being used remain to be established.

In conclusion, using GFP as a neoantigen for both in vivo and ex vivo methods of epidermal gene transfer, we show significant differences in the type of immune responses induced by two methods of gene transfer. Our data suggest that different strategies for suppression of destructive immune responses must be developed for ex vivo and in vivo gene therapy. Recent observations indicate that $\mathrm{Th} 2$ cells are more susceptible than Th1 cells to tolerance induction through blockade of costimulatory molecules, suggesting that the control of transgene-specific immune responses in the ex vivo method of gene therapy may be more manageable. ${ }^{35}$

\section{MATERIALS AND METHODS}

\section{Animals and retrovirus-mediated in vivo and ex vivo transduction of skin}

FVB mice were from Taconic Laboratories (James Town, NY). FVB-GadGFP and FVBGFPNagy transgenic lines were purchased from Jackson Laboratories (Bar Harbor, MA). GFP expression in the GFP-Nagy transgenic line is controlled by actin promoter and therefore expressed in all nucleated cell types, 36 whereas in Gad-GFP transgenic mice, expression is limited to hippocampal and cortical GABAergic interneurons in the brain.37 LZRS-based retroviral vectors 38 encoding GFP under the control of a $5^{\prime}$ long terminal repeat (LZRS-GFP) or truncated involucrin promoter (INV-GFP) were pseudotyped with vesicular somatitis virus envelope protein and used for transduction.20 For ex vivo gene transfer, epidermal cells isolated from newborn mouse skin (FVB) were cultured and 3 days later keratinocytes were transduced with LZRS-GFP at a multiplicity of infection of 2, resulting in $80 \%$ or higher transduction efficiencies. ${ }^{5}$ Transduced keratinocytes were harvested 3-4 days post-transduction to allow 
efficient depletion of dendritic cells.5,39 Epidermal cells $\left(2-3 \times 10^{6}\right)$ were combined with 4 $\times 10^{6}$ newborn dermal fibroblasts (isolated from FVB mice) and implanted as a slurry (150 $\mu$ l volume) onto the fascia but under a silicon chamber implanted onto the back of an anesthetized mouse. ${ }^{5}$ After 1 week, chambers were removed and wounds were allowed to heal. A well-differentiated skin appeared 7-10 days thereafter.

For in vivo transduction of cutaneous epithelium, the clipped, depilated dorsal skin of mice was dermabraded, and on day 3 post-abrasion $30 \mu \mathrm{l}$ (containing $3 \times 10^{7}$ transducing units) of INV-GFP or LZRS-GFP was used to transduce the healing skin as described previously. ${ }^{40}$ All strains of mice used in this study were males between 6 and 9 weeks of age at the time of initial gene transfer or transplantation. Males were used as graft recipients to avoid the malespecific $\mathrm{H}-\mathrm{Y}$ antigen-mediated rejection of primary epidermal keratinocytes isolated from newborns. Mice were housed individually and all procedures were performed under sterile conditions. Animal studies were performed in accordance with the institutional guidelines set forth by the Institute of Comparative Medicine at Columbia University.

\section{Detection of transgene expression in skin}

To assess GFP expression in the transduced skin of live animals, animals were anesthetized and placed under a fluorescent stereoscope (Bio 2M, Zeiss, Thornwood, NY) equipped with a mercury lamp and wide band filter set for GFP. Images were captured using a Nikon Coolpix 995 (Nikon Instrument, Melville, NY) and processed using Adobe Photoshop.

\section{Phenotypic analysis of infiltrating cells}

For histological evaluation of transduced skin tissues, biopsies were fixed in $10 \%$ phosphatebuffered formalin, embedded in paraffin, sectioned and stained with hematoxylin and eosin. To identify mast cells, sections were stained with $0.1 \%$ toluidine blue, which stains mast cells red-purple and the background blue. For B cell immunostaining, sections were incubated with 1:50 dilution of anti-B220 antibody (BD-Pharmingen, San Diego, CA). Bound antibodies were detected with a link-label IHC detection system (Biogenex, San Ramon, CA). For other immune markers, cryosections were prepared, fixed in acetone, blocked in 5\% non-fat milk in phosphate-buffered saline and stained with $1 \mu \mathrm{g} / \mathrm{ml}$ rat anti-mouse CD4, anti-mouse CD8a, or F4/80 monoclonal antibodies (BD-Pharmingen, San Diego, CA). Bound antibodies were detected using the Alexa 594-conjugated goat anti-rat antibody (Molecular Probes, Eugene, OR). Immunostained sections were mounted in Vectashield mounting media with DAPI (Vector Laboratories, Burlingame, CA), examined by a Zeiss Axioplan II fluorescent microscope and processed using Axiovision software (Zeiss, Thornwood, NY). Cellular infiltrates were assessed in four randomly selected fields at $\times 400$ original magnification.

\section{Analysis of humoral responses in transduced mice}

Antibody responses to GFP were determined by ELISA using recombinant GFP (BD Biosciences Clontech, Palo Alto, CA). Microtiter plates (Costar, Corning, NY) were coated with $100 \mathrm{ng}$ of recombinant GFP in carbonate buffer for $16 \mathrm{~h}$ at $4 \nmid \mathrm{C}$. The wells were washed and blocked with 5\% nonfat dry milk in tris-buffered saline for $1 \mathrm{~h}$ at room temperature. Diluted serum samples were added to the blocking solution and incubated for $2 \mathrm{~h}$. The wells were washed extensively, refilled with $100 \mu \mathrm{l}$ of 1:10,000 diluted horseradish peroxidase-conjugated goat anti-mouse IgG (Sigma, St Louis, MO), and bound antibodies were detected using tetramethyl-benzidine as substrate (Sigma). For antibody isotyping, after the 2-h incubation of serum samples, the identical sample wells were incubated with 1:1,000 dilution of goat antimouse IgG1, IgG2a, IgG2b, IgG3 (Sigma), or IgE (BD-Pharmingen, San Jose, CA) for 60 min followed by incubation with horseradish peroxidase-conjugated rabbit anti-goat antibodies before detection of the bound antibodies. 


\section{CTL assay}

Spleen and draining lymph nodes cells were isolated from transduced mice at 2 weeks following in vivo transduction or 4 weeks after transplantation of ex vivo-transduced keratinocytes and placed in culture for 5 days in the presence of mitomycin-treated GFPexpressing splenocytes isolated from FVB-Nagy-GFP mice. The stimulation ratio was five lymphocytes:one stimulator cell. Subsequently, stimulated lymphocytes (effector cells) were harvested, layered on Ficoll to remove dead cells, and mixed with syngeneic target cells (mouse GFP-expressing fibroblasts isolated from Nagy-GFP transgenic mice) in triplicate at different stimulator:target ratios (50:1 to 3:1) and incubated for $5 \mathrm{~h}$. LZ-transduced syngeneic fibroblasts were used as control targets. Specific lysis of target cells was determined with the LDH cytotoxicity detection kit following the manufacturer is protocols (Roche, Indianapolis, IN).

\section{Cytokines assay}

Cells from spleen and lymph nodes draining the transduced skin were used as responders $(5 \times$ $10^{6}$ well) and seeded with $2 \times 10^{6}$ mitomycin-treated GFP+splenocytes in 24-well plates for $72 \mathrm{~h}$. Cytokine concentration in the culture supernatants was determined by ELISA. Paired anti-mIFN- $\gamma$, anti-mIL-4, anti-mIL-5, and anti-mIL-2 monoclonal antibodies were purchased from BD-Pharmingen. Concentration was calculated based on a standard curve using recombinant mIL-4, mIFN- $\gamma$, mIL-2 (Peprotech, Rockhill, NJ), and mIL-5 (Ebioscience, San Diego, CA).

\section{In vivo anti-IL5 antibody treatment}

Functional-grade anti-mIL-5 antibody (TRFK5; rat IgG1 mAb) was purchased from Ebioscience. Mice were injected intraperitoneally with either $1 \mathrm{mg} / \mathrm{kg}(30 \mu \mathrm{g}$ in $100 \mu \mathrm{l}$ of sterile phosphate-buffered saline) of TRFK-5 or a control antibody (Rat IgG, Sigma) 1 day before transplantation of transduced keratinocytes. This treatment has been shown to inhibit successfully the release of eosinophils from bone marrow for at least 8 weeks and also to inhibit antigen-dependent lung eosinophilia in mice for at least 12 weeks. ${ }^{24}$

\section{Statistical analysis}

Data were expressed as the mean \pm SEM. Student's $t$ test was used in the analysis of the results and $P<0.05$ was considered significant.

\section{Acknowledgments}

We are grateful to Lorne B Taichman (Stony Brook University) for critically reviewing this manuscript and to Nancy Zhou (Columbia University) for technical assistance. This research was supported by Grant R01-AR050525 from National Institute of Health to SG.

\section{REFERENCES}

1. Ghazizadeh S, Katz AB, Harrington R, Taichman LB. Lentivirus-mediated gene transfer to human epidermis. J Invest Dermatol Symp Proc 2004;9:269-275.

2. Serrano F, Del Rio M, Larcher F, Garcia M, Munoz E, Escamez MJ, et al. A comparison of targeting performance of oncoretroviral versus lentiviral vectors on human keratinocytes. Hum Gene Ther 2003;14:1579-1585. [PubMed: 14577919]

3. Dellambra E, Pellegrini G, Guerra L, Ferrari G, Zambruno G, Mavilio F, et al. Toward epidermal stem cell-mediated ex vivo gene therapy of junctional epidermolysis bullosa. Hum Gene Ther 2000;11:2283-2287. [PubMed: 11084687]

4. Ghazizadeh S, Kalish RS, Taichman LB. Immune-mediated loss of transgene expression in skin: implications for cutaneous gene therapy. Mol Ther 2003;7:296-303. [PubMed: 12668125] 
5. Lu Z, Ghazizadeh S. Host immune responses in ex vivo approaches to cutaneous gene therapy targeted to keratinocytes. Exp Dermatol 2005;14:727-735. [PubMed: 16176280]

6. Brown BD, Lillicrap D. Dangerous liaisons: the role of "danger" signals in the immune response to gene therapy. Blood 2002;100:1133-1140. [PubMed: 12149189]

7. Song ES, Lee V, Surh CD, Lynn A, Brumm D, Jolly DJ, et al. Antigen presentation in retroviral vectormediated gene transfer in vivo. Proc Natl Acad Sci USA 1997;94:1943-1948. [PubMed: 9050884]

8. Cho JH, Youn JW, Sung YC. Cross-priming as a predominant mechanism for inducing CD8(+) T cell responses in gene gun DNA immunization. J Immunol 2001;167:5549-5557. [PubMed: 11698425]

9. Heath WR, Carbone FR. Cross-presentation, dendritic cells, tolerance and immunity. Annu Rev Immunol 2001;19:47-64. [PubMed: 11244030]

10. Anderson CC, Carroll JM, Gallucci S, Ridge JP, Cheever AW, Matzinger P. Testing time-, ignorance-, and danger-based models of tolerance. J Immunol 2001;166:3663-3671. [PubMed: 11238605]

11. Pasare C, Medzhitov R. Toll-like receptors: linking innate and adaptive immunity. Adv Exp Med Biol 2005;560:11-18. [PubMed: 15932016]

12. Ferrari S, Pellegrini G, Mavilio F, De Luca M. Gene therapy approaches for epidermolysis bullosa. Clin Dermatol 2005;23:430-436. [PubMed: 16023940]

13. Matzinger P. Tolerance, danger, and the extended family. Annu Rev Immunol 1994;12:991-1045. [PubMed: 8011301]

14. Heath WR, Belz GT, Behrens GM, Smith CM, Forehan SP, Parish IA, et al. Cross-presentation, dendritic cell subsets, and the generation of immunity to cellular antigens. Immunol Rev 2004;199:926. [PubMed: 15233723]

15. Rocha PN, Plumb TJ, Crowley SD, Coffman TM. Effector mechanisms in transplant rejection. Immunol Rev 2003;196:51-64. [PubMed: 14617197]

16. Yang Y, Ertl HC, Wilson JM. MHC class I-restricted cytotoxic T lymphocytes to viral antigens destroy hepatocytes in mice infected with E1-deleted recombinant adenoviruses. Immunity 1994;1:433-442. [PubMed: 7533647]

17. Tripathy SK, Black HB, Goldwasser E, Leiden JM. Immune responses to transgene-encoded proteins limit the stability of gene expression after injection of replication-defective adenovirus vectors. Nat Med 1996;2:545-550. [PubMed: 8616713]

18. Le Moine A, Goldman M, Abramowicz D. Multiple pathways to allograft rejection. Transplant 2002;73:1373-1381.

19. Le Moine A, Goldman M. Non-classical pathways of cell-mediated allograft rejection: new challenges for tolerance induction? Am J Transplant 2003;3:101-106. [PubMed: 12603204]

20. Ghazizadeh S, Doumeng C, Taichman LB. Durable and stratum-specific gene expression in epidermis. Gene Ther 2002;9:1278-1285. [PubMed: 12224010]

21. Abbas AK, Janeway CA Jr. Immunology: improving on nature in the twenty-first century. Cell 2000;100:129-138. [PubMed: 10647937]

22. Paul WE, Seder RA. Lymphocyte responses and cytokines. Cell 1994;76:241-251. [PubMed: 7904900]

23. Sanderson CJ. Interleukin-5, eosinophils, and disease. Blood 1992;79:3101-3109. [PubMed: 1596561]

24. Garlisi CG, Kung TT, Wang P, Minnicozzi M, Umland SP, Chapman RW, et al. Effects of chronic anti-interleukin-5 monoclonal antibody treatment in a murine model of pulmonary inflammation. Am J Respir Cell Mol Biol 1999;20:248-255. [PubMed: 9922215]

25. Schowalter DB, Meuse L, Wilson CB, Linsley PS, Kay MA. Constitutive expression of murine CTLA4Ig from a recombinant adenovirus vector results in prolonged transgene expression. Gene Ther 1997;4:853-860. [PubMed: 9338015]

26. Dobrzynski E, Fitzgerald JC, Cao O, Mingozzi F, Wang L, Herzog RW. Prevention of cytotoxic T lymphocyte responses to factor IX-expressing hepatocytes by gene transfer-induced regulatory $\mathrm{T}$ cells. Proc Natl Acad Sci USA 2006;103:4592-4597. [PubMed: 16537361]

27. Herzog RW, Dobrzynski E. Immune implications of gene therapy for hemophilia. Semin Thromb Hemost 2004;30:215-226. [PubMed: 15118933] 
28. Sanderson CJ. Interleukin-5: an eosinophil growth and activation factor. Dev Biol Stand 1988;69:2329. [PubMed: 3265676]

29. Braun MY, Desalle F, Le Moine A, Pretolani M, Matthys P, Kiss R, et al. IL-5 and eosinophils mediate the rejection of fully histoincompatible vascularized cardiac allografts: regulatory role of alloreactive CD8(+) T lymphocytes and IFN-gamma. Eur J Immunol 2000;30:1290-1296. [PubMed: 10820374]

30. Le Moine A, Flamand V, Demoor FX, Noel JC, Surquin M, Kiss R, et al. Critical roles for IL-4, IL-5, and eosinophils in chronic skin allograft rejection. J Clin Invest 1999;103:1659-1667. [PubMed: 10377172]

31. Surquin M, Le Moine A, Flamand V, Nagy N, Rombaut K, Demoor FX, et al. Skin graft rejection elicited by $\beta 2$-microglobulin as a minor transplantation antigen involves multiple effector pathways: role of fas-fas ligand interactions and Th2-dependent graft eosinophil infiltrates. J Immunol 2002;169:500-506. [PubMed: 12077281]

32. Agnello D, Lankford CS, Bream J, Morinobu A, Gadina M, O’Shea JJ, et al. Cytokines and transcription factors that regulate $\mathrm{T}$ helper cell differentiation: new players and new insights. J Clin Immunol 2003;23:147-161. [PubMed: 12797537]

33. Li M, Hener P, Zhang Z, Kato S, Metzger D, Chambon P. Topical vitamin D3 and low-calcemic analogs induce thymic stromal lymphopoietin in mouse keratinocytes and trigger an atopic dermatitis. Proc Natl Acad Sci USA 2006;103:11736-11741. [PubMed: 16880407]

34. Soumelis V, Reche PA, Kanzler H, Yuan W, Edward G, Homey B, et al. Human epithelial cells trigger dendritic cell mediated allergic inflammation by producing TSLP. Nat Immunol 2002;3:673-680. [PubMed: 12055625]

35. Kishimoto K, Dong VM, Issazadeh S, Fedoseyeva EV, Waaga AM, Yamada A, et al. The role of CD154-CD40 versus CD28-B7 costimulatory pathways in regulating allogeneic Th1 and Th2 responses in vivo. J Clin Invest 2000;106:63-72. [PubMed: 10880049]

36. Hadjantonakis AK, Gertsenstein M, Ikawa M, Okabe M, Nagy A. Generating green fluorescent mice by germline transmission of green fluorescent ES cells. Mech Dev 1998;76:79-90. [PubMed: 9867352]

37. Oliva AA Jr, Jiang M, Lam T, Smith KL, Swann JW. Novel hippocampal interneuronal subtypes identified using transgenic mice that express green fluorescent protein in GABAergic interneurons. J Neurosci 2000;20:3354-3368. [PubMed: 10777798]

38. Kinsella TM, Nolan GP. Episomal vectors rapidly and stably produce high-titer recombinant retrovirus. Hum Gene Ther 1996;7:1405-1413. [PubMed: 8844199]

39. Lei J, Cheng J, Li Y, Li S, Zhang L. CD80, but not CD86, express on cultured murine keratinocyte stem cells. Transplant Proc 2005;37:289-291. [PubMed: 15808622]

40. Ghazizadeh S, Harrington R, Taichman LB. In vivo transduction of mouse epidermis with recombinant retroviral vectors: implications for cutaneous gene therapy. Gene Ther 1999;6:12671275. [PubMed: 10455435] 

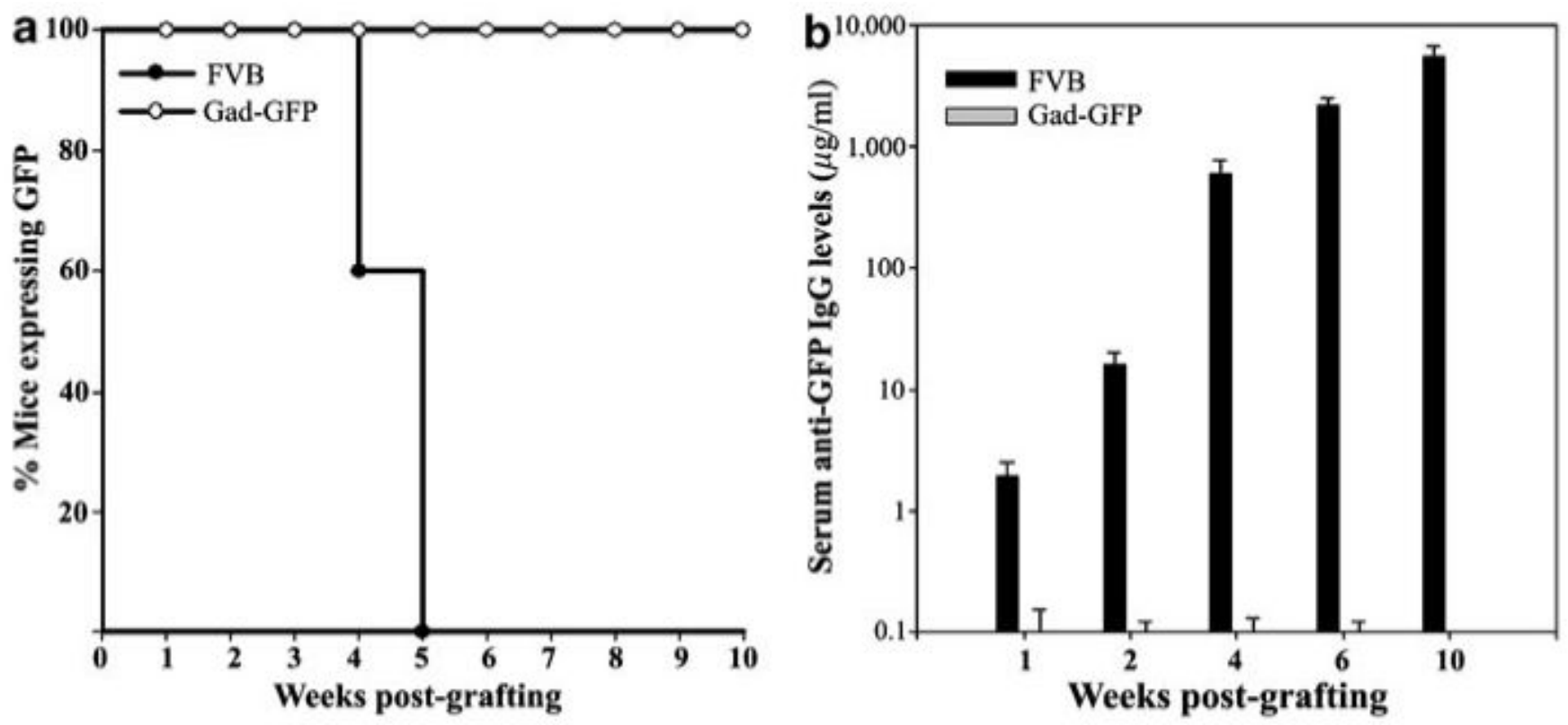

Figure 1. Kinetics of antigenic transgene loss and generation of anti-GFP antibody following $e x$ vivo cutaneous gene transfer

(a) Surface GFP in ex vivo-transduced skin of FVB mice was examined periodically by fluorescent stereoscopy until expression was lost ( $n=10)$. In FVB-Gad-GFP mice that are tolerant to GFP, transgene expression was long term and persisted throughout the observation period of 10 weeks $(n=6)$. (b) Sera was collected from ex vivo-transduced FVB or Gad-GFP mice at indicated times and analyzed for anti-GFP IgG by ELISA using a monoclonal antiGFP as a standard $(n=6)$. The increasing levels of IgG by time are shown in logarithmic scales. 

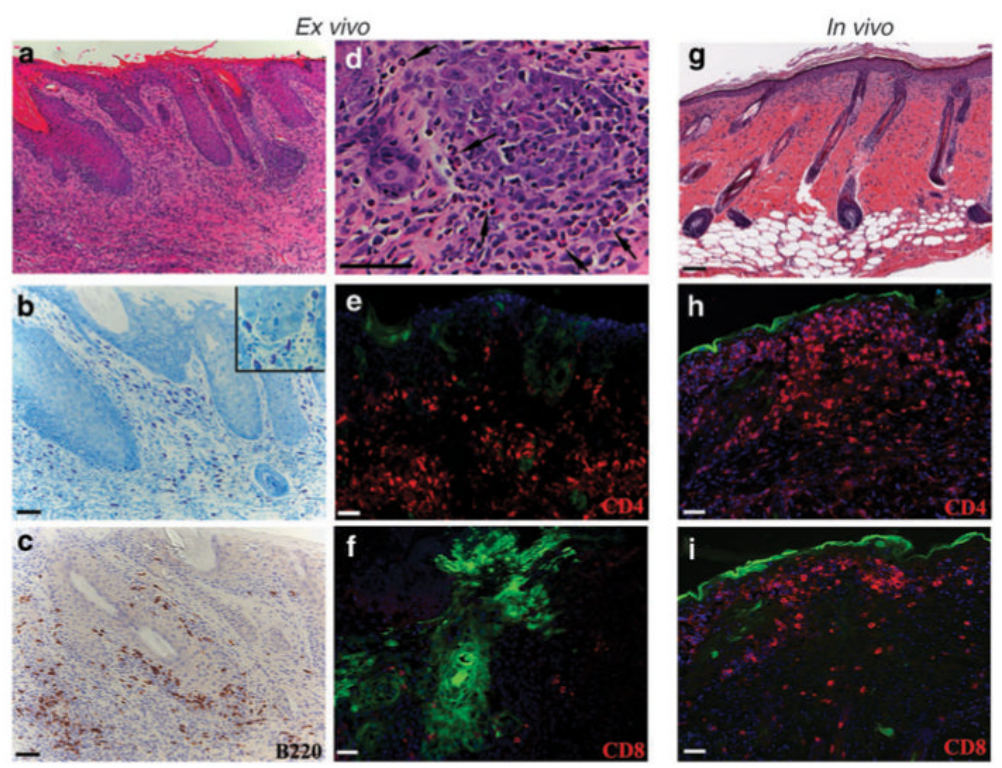

Figure 2. Cellular infiltrates in the ex vivo- and in vivo-transduced skin of mice

(a-f) Histological analysis of skin reconstituted from ex vivo-transduced keratinocytes on FVB mice at 4 weeks post grafting ( $\mathbf{g}-\mathbf{i})$ or that of in vivo-transduced skin at 2 weeks posttransduction. (a, d, g) Skin sections were stained with hematoxylin/eosin and showed pronounced dermal infiltration. (b) Skin sections were stained with toluidine blue to identify mast cells that appear red-purple in color. The image in $\mathbf{d}$ is at a higher magnification than that in showing numerous eosinophils (arrow) easily identified by their pluri-lobed nuclei and intense eosin staining of their cytoplasm. Sections were immunostained with antibodies recognizing B cells (B220), CD4+ cells (CD4), and cytotoxic T cells (CD8). (e, g, h, i)In sections stained by immunofluorescent, GFP-expressing cells are indicated by green and antibody staining in red. (h-i) GFP is about to be lost and expressed in the cornified layer in in vivo-transduced skin. Sections were counterstained with DAPI to visualize tissue structure and nuclei. Bar $100 \mu \mathrm{m}$ for $\mathbf{a}, \mathbf{g} ; 50 \mu \mathrm{m}$ for $\mathbf{b}-\mathbf{f}, \mathbf{h}, \mathbf{i}$. 

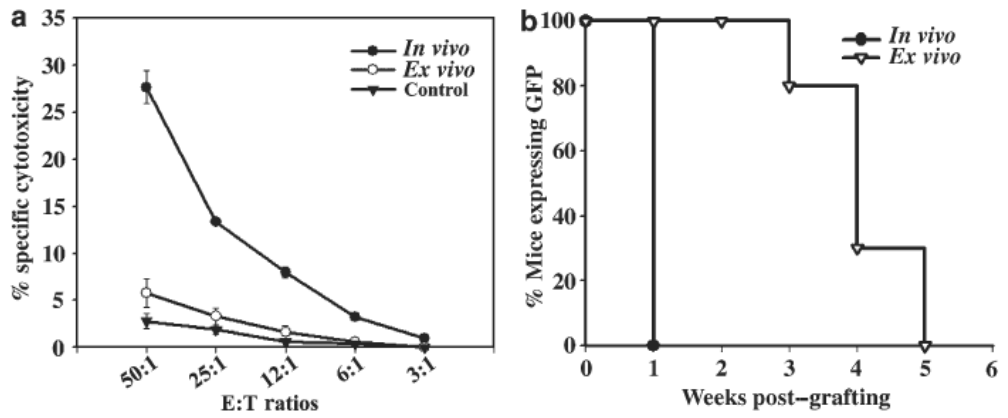

Figure 3. Analysis of GFP-specific CTL responses

(a) Stimulated lymphocytes (effector cells) were harvested and viable effector cells were mixed with GFP-expressing syngeneic fibroblasts at different effector:target ratios (50:1 to 3:1) and incubated for $5 \mathrm{~h}$. GFP-tolerant mice grafted with GFP+ keratinocyte were used as controls. Specific lysis of target cells was determined in triplicates using LZ-expressing syngeneic fibroblasts as control targets. (b) Mice were transduced in vivo $(n=4)$ with INV-GFP or ex vivo (n=6) with LZRS-GFP, and at 7 weeks posttransduction when GFP expression was lost in all mice, they were challenged with transplantation of GFP expressing keratinocytes. Mice were monitored weekly and GFP-expression in the grafted area was scored. A $\geq 90 \%$ decrease in surface GFP was taken to indicate graft loss. 

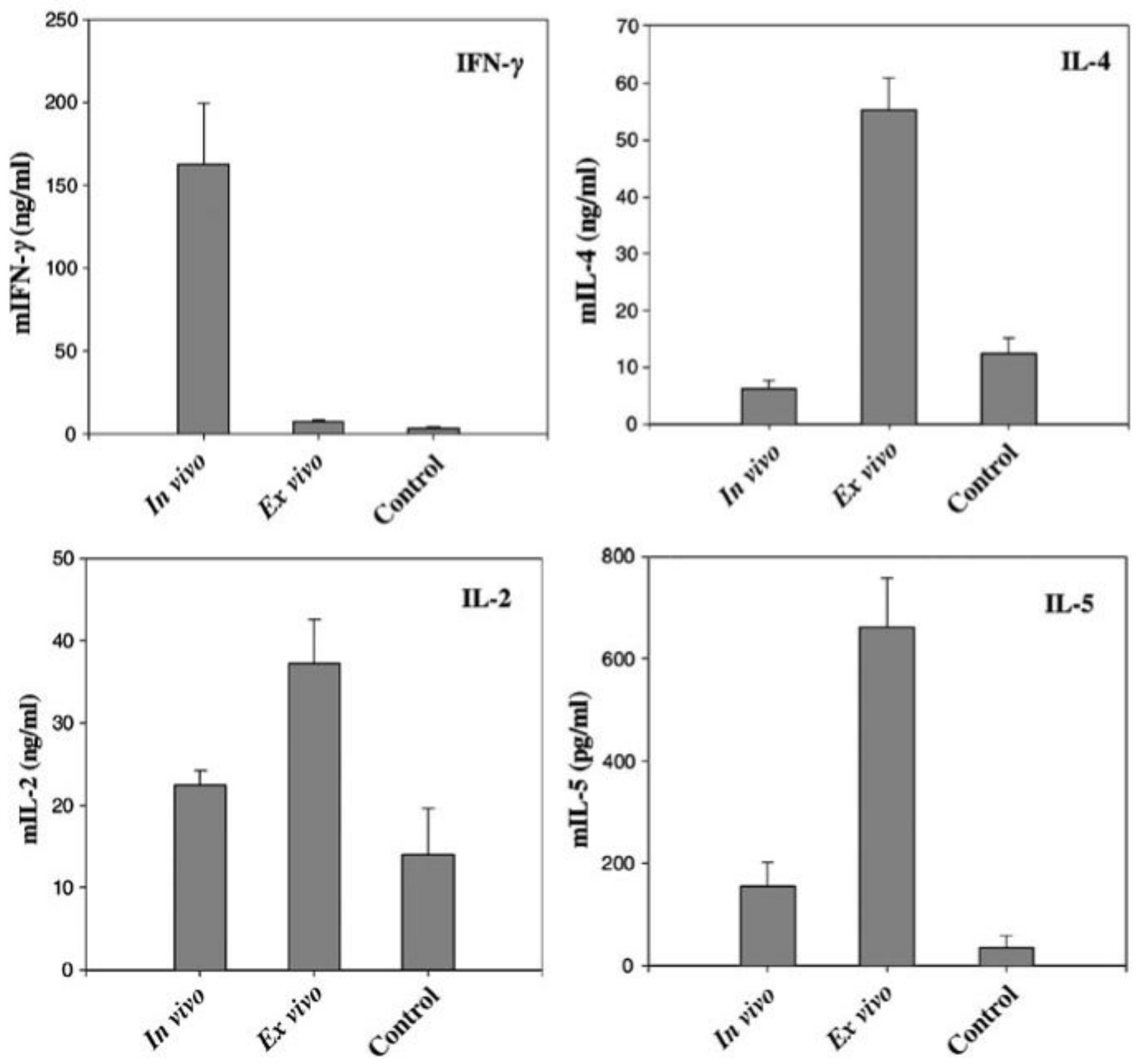

Figure 4. In vitro cytokine secretion by lymphocytes of transduced mice Splenocytes and lymphocytes from axillary lymph nodes were isolated at 2 or 5 weeks posttransduction from in vivo- or ex vivo-transduced mice, respectively. Controls are Gad-GFP mice transplanted with GFP+ keratinocytes. Lymphocytes were cultured with mitomycintreated GFP-expressing splenocytes for $72 \mathrm{~h}$. Cell-free supernatant was collected and analyzed by cytokine-specific ELISA as indicated in the graph $(n=6)$. Results are expressed as mean \pm SEM. 

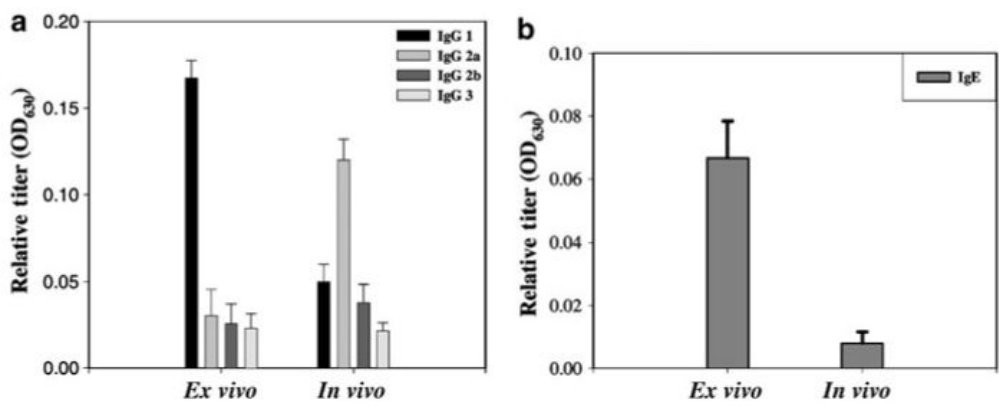

Figure 5. Analysis of GFP-specific antibody responses in transduced mice

Sera were collected from in vivo- or ex vivo-transduced mice at 4 weeks posttransduction and assayed for the presence of anti-GFP IgG subtypes by ELISA using goat antibodies specific for (a) mouse IgG1, IgG2a, IgG2b, or IgG3 or (b) for IgE. Data are expressed as the means of the optical density at $630 \mathrm{~nm}\left(\mathrm{~A}_{630}\right) \pm \mathrm{SEM}$ for $1: 100$ dilution of the serum samples $(n=6)$. 

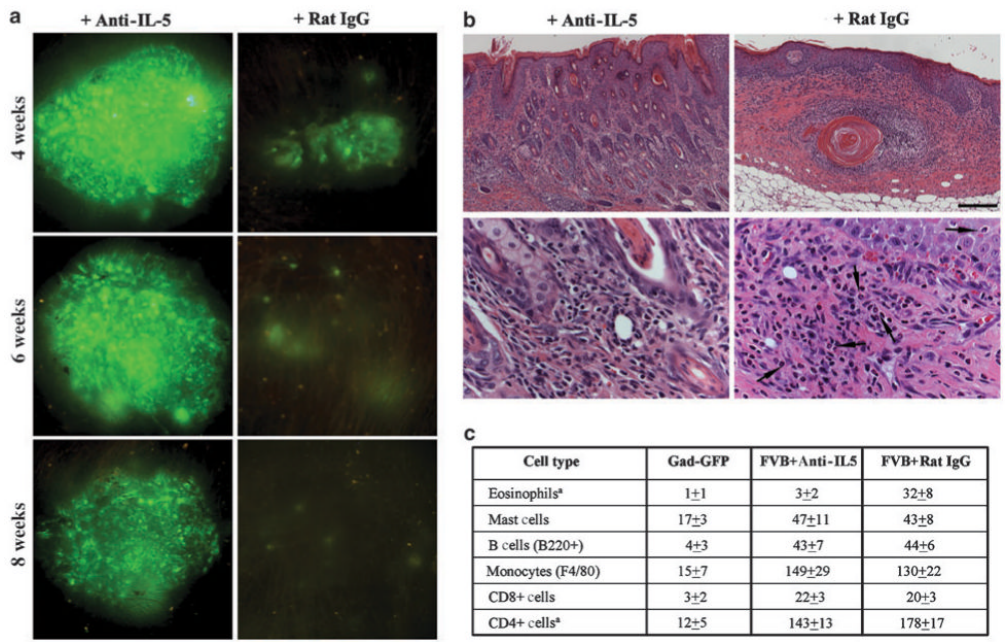

Figure 6. Effect of IL-5blockade on transgene expression in ex vivo-transduced mice

(a) Prolonged surface GFP expression in a representative mouse receiving a single injection of $30 \mu \mathrm{g}$ of anti-mIL5 (TRFK5) followed by grafting of ex vivo-transduced keratinocytes. In mice pretreated with $30 \mu \mathrm{g}$ of control rat IgG, surface GFP is almost lost at 4 weeks postgrafting. (b) Histological analysis of transduced skin sections obtained at 4 weeks posttransduction and stained with hematoxylin/eosin staining shows suppression of eosinophil infiltration in mice treated with anti-IL5. Lower panels are at higher magnification to show eosinophilic infiltrates. Eosinophils (arrows) are easily identified by their plurilobed nuclei and the intense eosin staining of their cytoplasm. (c) Summary of distribution of inflammatory cells in skin reconstituted on GFP-tolerant mice or FVB mice pretreated with anti-IL-5 antibody or RatIgG. Cellular infiltrates were assessed in four randomly selected fields at $\times 400$ original magnification and are expressed as mean \pm SEM. 